\title{
Tumor suppressor FOXO3 participates in the regulation of intestinal inflammation
}

\author{
Lobke Snoeks ${ }^{1}$, Christopher R Weber ${ }^{2}$, Kaarin Wasland ${ }^{1}$, Jerrold R Turner $^{2}$, Charles Vainder ${ }^{1}$, Wentao Qi ${ }^{1}$ \\ and Suzana D Savkovic ${ }^{1}$
}

Inflammatory bowel disease (IBD), including Crohn's disease and ulcerative colitis, is characterized by chronic mucosal injury and the infiltration of inflammatory cells. Tumor suppressor FOXO3 regulates gene expression and its translocation to the cytosol leads to the abrogation of its transcriptional function. We have previously shown that bacterial infection regulates $\mathrm{FOXO} 3$ in intestinal epithelial cells and increases cytokine levels. As TNF $\alpha$ is a major contributor in intestinal inflammation, the aim of this study was to assess its effect on FOXO3 and FOXO3's contribution to intestinal inflammation in vitro and in vivo. TNF $\alpha$ induces the translocation of nuclear $\mathrm{FOXO} 3$ into the cytosol where it undergoes proteasomal degradation in human intestinal HT-29 cells. Proximally, the PI3K and IKK pathways mediate TNF $\alpha$-induced FOXO3 phosphorylation. In FOXO3-silenced HT-29 cells, TNF $\alpha$-induced IL-8 expression is increased $\sim 83 \%$. In vivo, Foxo3 is present in the nuclei and cytosol of colonic crypt epithelia. In DSS-induced colonic inflammation, Foxo3's nuclear localization is lost and it is only found in the cytosol. Consistent with a role for Foxo3 in colitis, Foxo3-deficient mice treated with DSS developed more severe colonic inflammation with an increased number of intraepithelial lymphocytes and PMNs infiltrated in the epithelia, than wild-type mice. In summary, TNF $\alpha$ inactivates FOXO3 in intestinal epithelia through the PI3K and IKK pathways and FOXO3 inactivation leads to the upregulation of IL-8 in vitro; in vivo Foxo3 is in the cytosol of inflamed colonic epithelia and Foxo3 deficiency leads to severe intestinal inflammation.

Laboratory Investigation (2009) 89, 1053-1062; doi:10.1038/labinvest.2009.66; published online 27 July 2009

KEYWORDS: FOXO3; inflammation; intestinal epithelia; signaling; TNF $\alpha$

In the intestinal tissue of inflammatory bowel disease (IBD) patients with the active disease, many proinflammatory cytokines are increased, which are imperative in maintaining inflammatory responses. ${ }^{1}$ One of the cytokines that drives intestinal inflammation is tumor necrosis factor- $\alpha(\mathrm{TNF} \alpha){ }^{2-4}$ TNF $\alpha$-blocking agents significantly reduce intestinal inflammation in the majority of patients with active IBD. ${ }^{5}$

$\mathrm{TNF} \alpha$ binds to cell membrane TNF $\alpha$ receptors (TNFR1 and TNFR2), ${ }^{6-8}$ stimulating signaling cascades that lead to the activation of nuclear factor kappaB $(\mathrm{NF}-\kappa \mathrm{B}){ }^{9}$ From TNFRs, the intracellular signaling pathways, that include several receptor adapters, activate the inhibitory kappaB $(\mathrm{I} \kappa \mathrm{B})$ kinase $(\mathrm{IKK})$ complex. Activated IKK phosphorylates $\mathrm{I} \kappa \mathrm{B}$ proteins causing $\mathrm{I} \kappa \mathrm{B}$ degradation and the release of $\mathrm{NF}-\kappa \mathrm{B}$ to freely translocate to the nucleus. ${ }^{10}$ Nuclear NF- $\kappa \mathrm{B}$ regulates genes that are involved in inflammation, cell survival, and proliferation. ${ }^{11,12}$
The tumor suppressor FOXO3 belongs to the family of Forkhead transcriptional factors. ${ }^{13}$ FOXO3 is located in the nucleus regulating the expression of specific target genes involved in the regulation of cell cycle progression, metabolic state, and cellular apoptosis. ${ }^{13,14}$ Phosphorylation of FOXO3 can occur by upstream pathways that include PI3K and/or IKK $;^{14,15}$ phosphorylated FOXO3 translocates to the cytosol and becomes inactive. ${ }^{16}$ Cytosolic FOXO3 might attach to 14-3-3 proteins or be degraded in the proteasome. ${ }^{15-19}$

Foxo3-deficient mice develop spontaneous, multisystemic inflammatory syndrome, accompanied by an increased cytokine production, an increased NF- $\kappa \mathrm{B}$ activation, and hyperactivation of $\mathrm{T}$ cells. ${ }^{20}$ In $\mathrm{T}$ cells, the overexpressed Foxo 3 inhibits NF- $\kappa$ B and cytokine expression. ${ }^{20}$ We have demonstrated that bacterial infection inactivates FOXO3 in the intestinal epithelia and that this contributes to an increased cytokine expression; ${ }^{21}$ hence, we hypothesize

\footnotetext{
'Division of Gastroenterology, Department of Medicine, North Shore University Research Institute, Evanston, IL, USA and ${ }^{2}$ Department of Pathology, University of Chicago, Chicago, IL, USA

Correspondence: Dr SD Savkovic PhD, Department of Medicine, Division of Gastroenterology, NorthShore University Research Institute, 1001 University Place; Room 314, Evanston, IL 60201, USA.

E-mail: SSavkovic@northshore.org

Received 13 November 2008; revised 24 April 2009; accepted 12 May 2009
} 
that $\mathrm{FOXO} 3$ is involved in the regulation of intestinal inflammation. We have shown here that $\mathrm{TNF} \alpha$ inactivates FOXO3 in HT-29 cells through the PI3K and IKK pathways, which additionally increase IL-8. In mice, where colonic inflammation was induced with dextran sulfate sodium (DSS), Foxo3 is also inactive. Foxo3-deficient mice, in response to DSS, developed more severe intestinal inflammation compared with the wild-type mice. Altogether, our results suggest that tumor suppressor $\mathrm{FOXO} 3$ has an important role in the regulation of intestinal inflammation in vitro and in vivo.

\section{MATERIALS AND METHODS Tissue Culture}

Human intestinal epithelial HT-29 cells (ATCC, Manassas, VA, USA; passages 10-20) were cultured in complete McCoy's 5A media (Sigma-Aldrich, St Louis, MO, USA) containing 10\% fetal bovine serum (Gibco, Carlsbad, CA, USA) and Penicillin-Streptomycin (Gibco). The cells were kept in an incubator at $37^{\circ} \mathrm{C}$, with $5 \% \mathrm{CO}_{2}$ and then they were plated in 6- or 12-well plates. When the monolayers reached confluency of $\sim 70 \%$, cells were serum-starved overnight before the experimental procedures were performed.

Semi-confluent HT-29 monolayers were stimulated with $10 \mathrm{ng} / \mathrm{ml} \mathrm{TNF} \alpha(\mathrm{R} \& \mathrm{D}$, Minneapolis, MN, USA) for different lengths of time to study the effect on FOXO3 and IL-8.

\section{Immunofluorescent Staining}

For immunofluorescent staining, HT-29 cells were grown on coverslips. After treatment, the cells were washed with PBS and fixed with $3.7 \%$ paraformaldehyde. The fixed cells were washed with PBS and permeabilized with $0.2 \%$ Triton-X in PBS followed by blocking in $2.5 \%$ bovine serum albumin. The cells were incubated with a primary $\mathrm{FOXO} 3$ antibody (Upstate, Billerica, MA, USA) for $1 \mathrm{~h}$, followed by washing and incubation with a secondary antibody for $1 \mathrm{~h}$ (Alexa Fluor 488 Molecular Probes, Eugene, OR, USA). The coverslips with cells were mounted on microscope slides (Mounting Solution antifade reagent, Molecular Probes). The results were assessed using a Nikon Opti-Photo microscope. The images were captured using a Spot RT-slider camera (Diagnostic Instruments, Sterling Heights, MI, USA) and managed by Image Pro software (Media Cybernetics, San Diego, CA, USA).

\section{Inhibitor Studies}

To study different pathways in FOXO3 regulation, several pharmacological inhibitors were applied. To inhibit the IKK pathway, we utilized its specific inhibitor PS1145 $(30 \mu \mathrm{M})$ (Sigma). To study the role of the PI3K pathway inhibitors, wortmannin $(200 \mathrm{nM})$ (Calbiochem, Gibbstown, NJ, USA) and LY294002, $(30 \mu \mathrm{M})$ (Calbiochem) were used. The proteasome inhibitor MG132 $(20 \mu \mathrm{M})($ Calbiochem) was applied to study $\mathrm{FOXO} 3$ proteasome-dependent degradation. The serum-starved HT-29 monolayers were pre-incubated with an inhibitor for $1 \mathrm{~h}$ after which the monolayers were treated with $\mathrm{TNF} \alpha$ for different lengths of time.

\section{Protein Extraction}

After treatment, the HT-29 monolayers were washed with PBS (Gibco) and protein was extracted by applying cell lysis buffer (Cell Signaling, Danvers, MA, USA), containing a protease inhibitor cocktail (Sigma). Protein concentrations were determined using the Quick Start Bradford Protein Assay kit (Biorad, Hercules, CA, USA) as described by the manufacturer. The protein extracts were stored at $-20^{\circ} \mathrm{C}$ until further processing. The nuclear proteins were extracted using the NE-PER Pierce extraction kit (Pierce, Rockford, IL, USA) according to the manufacturer's protocol and stored at $-80^{\circ} \mathrm{C}$.

\section{Immunoblots}

Equal amounts of protein $(40 \mu \mathrm{g})$ per sample were separated on $10 \%$ Sodium Dodecyl Sulfate polyacrylamide (SDS-PAGE) gels. Protein was transferred to nitrocellulose membranes (Biorad). The immunoblots were probed with appropriate antibodies against $\mathrm{FOXO} 3$ (Upstate), antiphospho-Thr32-FOXO3 (Upstate), anti-phospho-Ser644FOXO3 (generous gift of $\mathrm{Dr} \mathrm{Hu}$ ), and actin (Santa Cruz Biotechnology, Santa Cruz, CA, USA) all according to the manufacturers' protocols. The secondary antibodies were horseradish peroxidase-conjugated (Chemicon, Temecula, CA, USA). The protein bands were detected by using the ECL Plus western blotting detection system (GE Healthcare, Amersham, UK).

\section{SiRNA Experiments}

To study the effects of FOXO3 on TNF $\alpha$-induced IL- 8 in intestinal epithelial cells, we transiently knocked-out FOXO3 by applying siRNA. The HT-29 cells were transfected with two different oligos, designed to silence FOXO3 $(30 \mathrm{nM})$ or a negative control oligo (Invitrogen, Carlsbad, CA, USA). Oligos were introduced into the cells using Lipofectamine RNAiMax (Invitrogen) following the manufacturers protocol. Initially, $48 \mathrm{~h}$ post-transfection showed optimal knock down of FOXO3. Therefore, $36 \mathrm{~h}$ after transfection with siRNA, the monolayers of HT-29 cells were serum deprived overnight and sequentially treated with TNF $\alpha$ for $4 \mathrm{~h}$. The media was collected and stored at $-20^{\circ} \mathrm{C}$ until it was used for IL-8 quantification. Whole cell lysates were used as a control for knock down of FOXO3 with western blotting.

\section{IL-8 Quantification}

To quantify IL-8, the media collected from monolayers was used for IL-8 ELISA (R\&D) according to the manufacturer's protocol. 


\section{Statistical Analysis}

These data were represented as the mean \pm s.d.. The student's $t$-test was used to compare these data and the differences were considered significant when the $P$-values were $\leq 0.05$.

\section{Animal Studies}

For an in vivo study, colonic inflammation was induced by introducing 2.5\% DSS (MP Biomedicals, LLS, Solon, OH, USA) into the drinking water of C57BL/6J mice (6-8 weeks old) for 5 days, followed by two recovery days. Body weight was monitored during the course of the treatment. Gastrointestinal bleeding was evaluated using Coulter Hemoccult (Fisher, Pittsburgh, PA, USA). After 7 days, the animals were euthanized and the colonic tissue was prepared for histopathological studies.

Furthermore, to study the in vivo role of $\mathrm{FOXO} 3$ in colonic inflammation, we utilized 4 to 6-week-old Foxo3-deficient mice kindly provided by Dr Stanford Peng (Roche Palo Alto LLC, Palo Alto, CA, USA). The genotypes of the breeds were determined by PCR on tail DNA using primers according to Lin et al. ${ }^{20}$

All animals were kept in the Biological Resources Laboratory at the University of Illinois at a Chicago facility and all experimental procedures were performed in compliance with the local protocols and guidelines approved by the local ethical committee.

\section{Histological Analysis}

Colonic tissue was formalin-fixed and paraffin-embedded. The tissue sections $(5-\mu \mathrm{m}$ thick) were stained with routine hematoxylin and eosin (H\&E) staining. The distribution of Foxo3 in colonic tissue was assessed as we described before. ${ }^{21}$ The degree of inflammation was evaluated according to the following criteria: (0) completely uninvolved, no architectural distortion or infiltrates; (1) architectural distortion, increased lamina propria lymphs, no activity; (2) increased lamina propria granulocytes without definite intraepithelial granulocytes (ie, no activity); (3) intraepithelial granulocytes (ie, activity) without crypt abscesses; (4) crypt abscesses in less than $50 \%$ of crypts; (5) crypt abscesses in greater than $50 \%$ of crypts or erosion/ulceration.

\section{RESULTS}

\section{TNF $\alpha$ Regulates FOXO3 in HT-29 Cells}

Nuclear FOXO3 is involved in the regulation of transcription; signaling by growth factors and other stimuli results in FOXO3 translocation from the nucleus to the cytosol and terminates $\mathrm{FOXO} 3$ gene regulation. ${ }^{16-18}$ This process can be modeled in human colonic epithelial HT-29 cells, where FOXO3 is localized in the nucleus but translocated to the cytosol after bacterial infection. ${ }^{21}$ To address the effect of cytokines on this process, FOXO3 localization was examined in HT-29 cells treated with TNF $\alpha$. FOXO3 translocated from the nucleus to the cytosol during the first $30 \mathrm{~min}$ of TNF $\alpha$ treatment (Figure 1a). An immunoblot analysis of subcellular fractions confirmed that the amount of nuclear FOXO3 decreases, whereas the amount of cytosolic FOXO3 increases after $\mathrm{TNF} \alpha$ stimulation (Figure $1 \mathrm{~b}$ ). This data suggests that FOXO3 becomes inactive after TNF $\alpha$ treatment of the HT-29 cells.

It was shown that cytosolic FOXO3 might degrade by proteasome. ${ }^{15}$ Therefore, we examined the fate of cytosolic FOXO3 in HT-29 cells treated with TNF $\alpha$. Figure 2a shows that TNF $\alpha$ induces FOXO3 degradation in colonic HT-29 cells. To further define the mechanism of TNF $\alpha$-induced FOXO3 degradation, we used the proteasome inhibitor MG132. The pretreatment of HT-29 cells with MG132 caused a small, statistically insignificant, increase in total FOXO3 and treatment with TNF $\alpha$ of MG132-pretreated HT-29 cells, blocked FOXO3 degradation (Figure $2 \mathrm{~b}$ ). In summary, TNF $\alpha$ induced the degradation of $\mathrm{FOXO} 3$ and that the degradation is proteasome mediated in HT-29 cells.

\section{TNF $\alpha$ Regulates FOXO3 in HT-29 Cells via PI3K and IKK}

The translocation of FOXO3 from the nucleus to the cytosol occurs after its phosphorylation. ${ }^{16}$ The PI3K pathway mediates the phosphorylation of FOXO3 in many cell types. ${ }^{16} \mathrm{We}$ have previously reported that LPS-induced FOXO3 phosphorylation in intestinal epithelial cells is through the PI3K pathway. ${ }^{21}$ Therefore, we assessed the role of PI3K in the phosphorylation of FOXO3 in intestinal epithelial cells treated with TNF $\alpha$. We used an antibody that recognizes FOXO3 phosphorylation at Thr32, which is known to be phosphorylated by the PI3K pathway. ${ }^{16}$ TNF $\alpha$ increased FOXO3 phosphorylation at Thr32 by $3.1 \pm 0.8$-fold in the first $30 \mathrm{~min}$ of treatment (Figure 3). The pharmacological PI3K inhibitors, wortmannin or LY294002, were used to assess the contribution of the PI3K pathway in TNF $\alpha$-induced FOXO3 phosphorylation. The inhibition of the PI3K pathway with these inhibitors blocked the phosphorylation of Akt, which is responsible for the phosphorylation of $\mathrm{FOXO}^{13}$ (data not shown). Both inhibitors blocked TNF $\alpha$-induced FOXO3 phosphorylation in HT-29 cells (Figure 3 shows inhibition with LY294002). Also, the inhibition of PI3K-blocked TNF $\alpha$ induced degradation of Foxo3 (data not shown). This data shows that TNF $\alpha$-induced FOXO3 phosphorylation and degradation in HT-29 cells is controlled proximally by the PI3K pathway.

$\mathrm{Hu}$ reported that $\mathrm{FOXO} 3$ might be phosphorylated by IKK in breast cancer cells. ${ }^{15}$ To examine the contribution of IKK in TNF $\alpha$-dependent FOXO3 inactivation we employed an antibody that recognizes FOXO3 phosphorylation at Ser644, which is only phosphorylated by IKK. ${ }^{15}$ The $\mathrm{TNF} \alpha$ treatment of HT-29 cells increased FOXO3 Ser644 phosphorylation $2.7 \pm 0.2$-fold within $30 \mathrm{~min}$ (Figure 4a). To further examine the role of the IKK pathway in TNF $\alpha$ induced FOXO3 regulation in HT-29 cells, we used a specific IKK-inhibitor, PS1145. ${ }^{22,23}$ We confirmed the specificity of IKK inhibition by PS1145 by showing the attenuation of p65 phosphorylation ${ }^{24}$ (data not shown). Pretreatment 
a
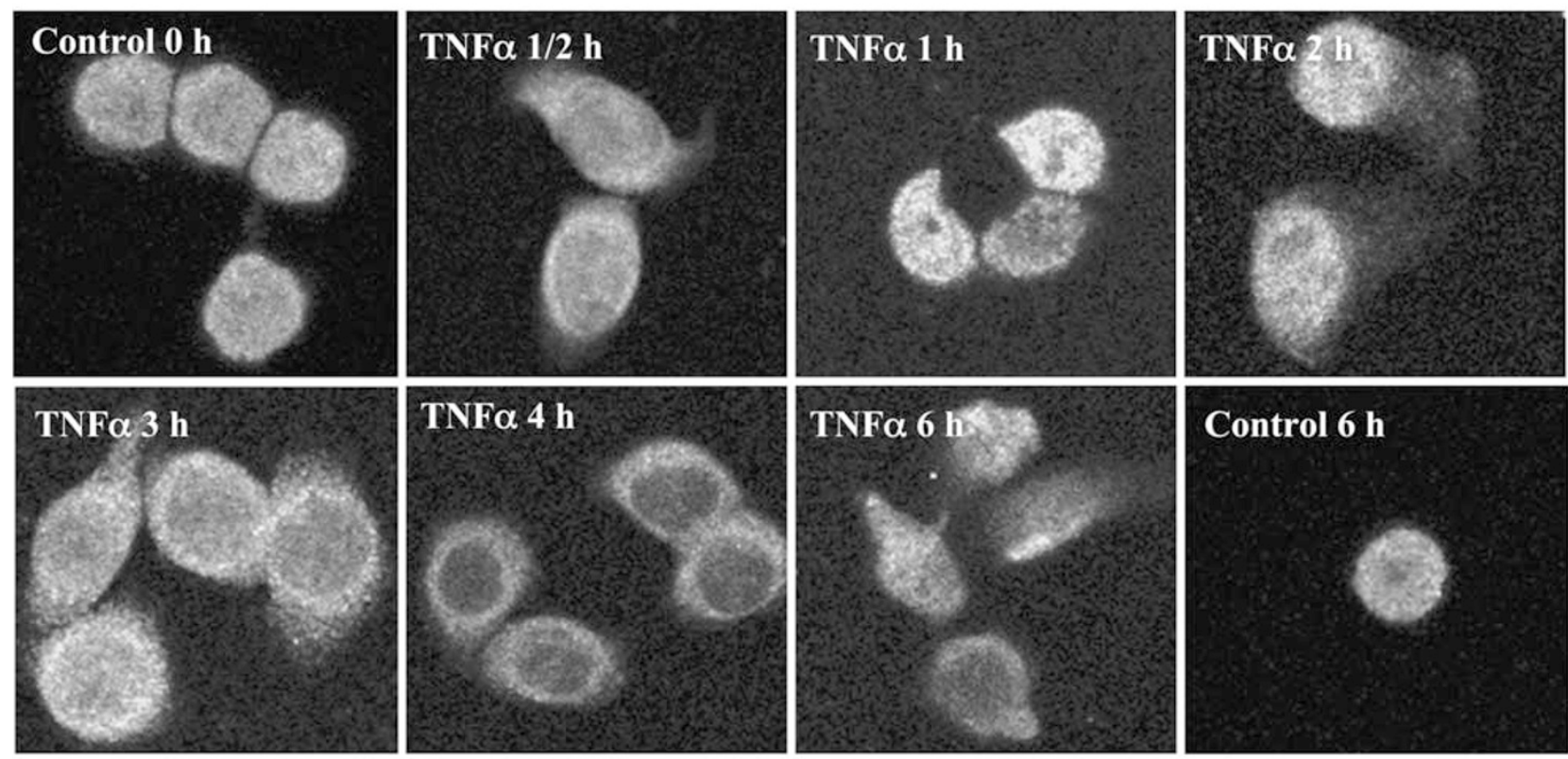

b
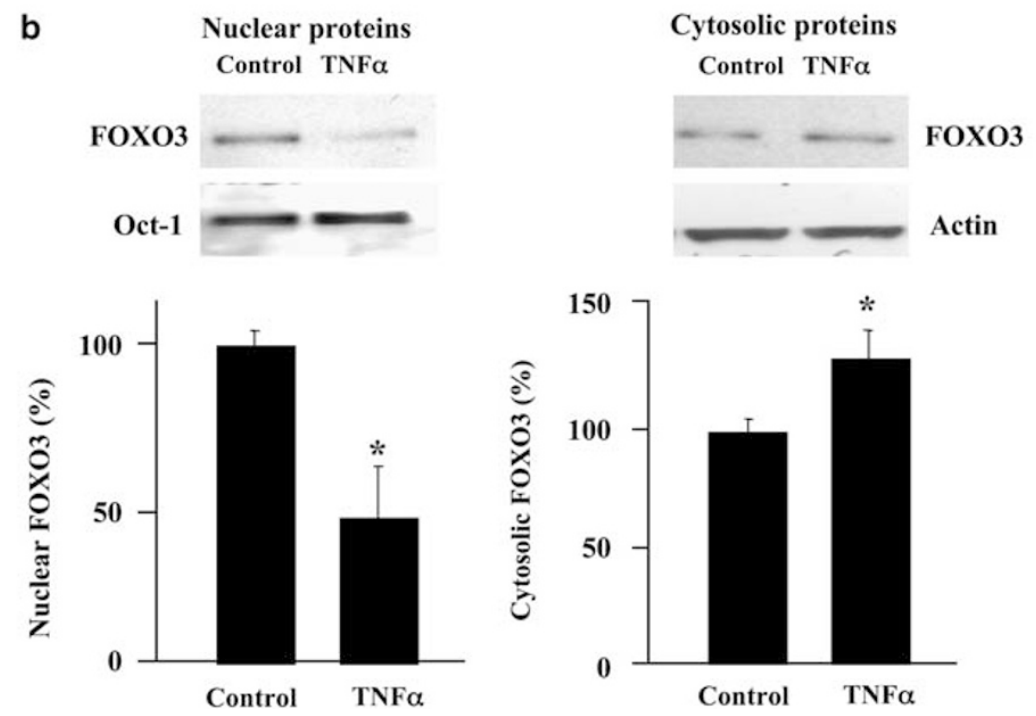

Figure 1 TNF $\alpha$ induces FOXO3 translocation in HT-29 cells. (a) HT-29 cells, control and treated with TNF $\alpha$ were fixed, immunofluorescent stained for FOXO3 and images were taken with matched exposures. In control cells, FOXO3 is localized in the nucleus. During TNF $\alpha$ treatment, nuclear FOXO3 translocates to the cytosol, suggesting its inactivation ( $\times 60$ magnification). This experiment was repeated three independent times. (b) Nuclear ( $8 \mu \mathrm{g})$ and cytosolic ( $40 \mu \mathrm{g}$ ) proteins from HT-29 cells, control and TNF $\alpha$ treated for $3 \mathrm{~h}$, were separated on SDS-PAGE and immunoblotted for FOXO3. Immunoblots reveal decreased nuclear and increased cytosolic amounts of FOXO3 after TNF $\alpha$ treatment. Immunoblots were reprobed with antibodies against Oct-1 for nuclear extracts and actin for cytosolic extracts as a control. Densitometric analysis shows a significant differences $\left({ }^{*}\right)$ between groups $(P<0.05)$.

of HT-29 cells with PS1145 almost completely blocked TNF $\alpha$-induced FOXO3 degradation (Figure 4b). These data suggest that IKK participates in the regulation of FOXO3 in HT-29 cells treated with TNF $\alpha$. The inhibition of IKK did not change PI3K-dependent phosphorylation of $\mathrm{FOXO} 3$ (Figure 4c), suggesting that $\mathrm{FOXO} 3$ regulation by PI3K is independent of IKK in HT-29 cells treated with TNF $\alpha$.
FOXO3 is Involved in the Regulation of TNF $\alpha$-Induced IL-8 IL-8 is a proinflammatory chemokine that is a strong chemoattractant for neutrophils and lymphocytes. ${ }^{25-27}$ In the tissue of IBD patients, IL-8 is significantly upregulated ${ }^{27-30}$ and the level of IL- 8 is in direct proportion to the degree of inflammation. ${ }^{30}$ Cultured intestinal epithelial cells secrete IL-8 following TNF $\alpha$ treatment. ${ }^{31,32}$ Under the conditions used in this study, HT-29 cells increased IL-8 secretion 

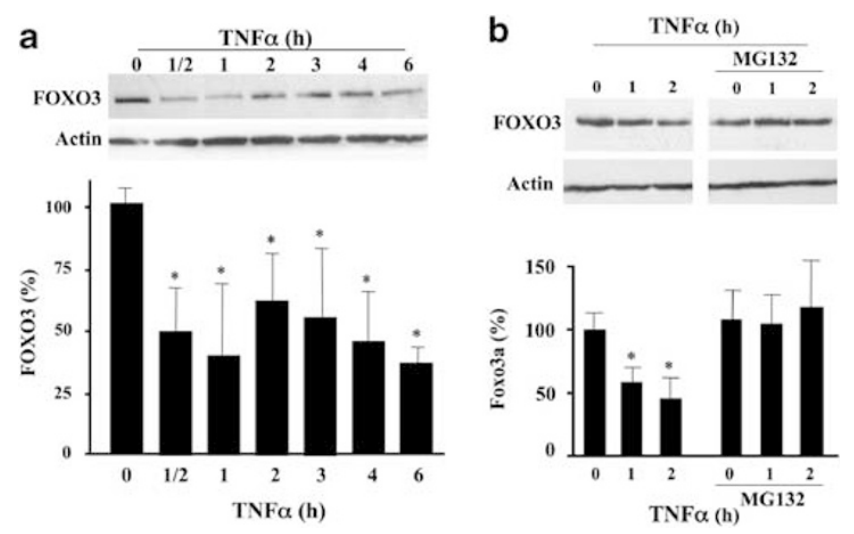

Figure $2 \mathrm{TNF} \alpha$ treatment of HT-29 cells induces degradation of FOXO3 by proteasome. (a) The total proteins from HT-29 cells, control and treated with TNF $\alpha$ for various time points, were separated on SDS-PAGE and immunoblotted for FOXO3 and actin. (b) The HT-29 monolayers were preincubated with proteasome inhibitor MG132 for $1 \mathrm{~h}$ and then treated with $\mathrm{TNF} \alpha$ for various time points. Protein was separated on SDS-PAGE and immunoblotted for $\mathrm{FOXO} 3$ and actin. Each experiment was performed in triplicate and the densitometric analysis shows significant ${ }^{*}$ ) degradation of FOXO3 during the course of TNF $\alpha$ treatment compared with untreated cells and protection with MG132 $(P<0.05)$.

20 -fold within $6 \mathrm{~h}$ of TNF $\alpha$ treatment (control: $23 \pm 2 \mathrm{pg} / \mathrm{ml}$; $\mathrm{TNF} \alpha: 456 \pm 13 \mathrm{pg} / \mathrm{ml}$ ) (Figure 5a). To assess the contribution of FOXO3 to TNF $\alpha$-induced IL- 8 expression in intestinal HT-29 cells, we performed siRNA experiments. The efficiency of FOXO3 silencing by siRNA in HT-29 cells was $~ 90 \%$ (Figure 5b). The silencing of FOXO3 increased the basal level of IL-8 in HT-29 monolayers insignificantly. IL-8 was increased on average $83 \%$ in monolayers with silenced FOXO3 compared with IL-8 in monolayers with negative siRNA control treated with $\mathrm{TNF} \alpha$ for $4 \mathrm{~h}(\mathrm{TNF} \alpha(-)$ control: $304 \pm 18 \mathrm{pg} / \mathrm{ml}$; TNF $\alpha$ siRNA: $544 \pm 110 \mathrm{pg} / \mathrm{ml}$ ) (Figure 5c). This data is consistent with our previously published data where an inactive $\mathrm{FOXO} 3$ contributes to the upregulation of IL-8 during bacterial infection. ${ }^{21}$

\section{Cytosolic FOXO3 is Associated with Colonic Inflammation}

To further examine the role of $\mathrm{FOXO} 3$ in in vivo intestinal inflammation, we used a mouse model. Mouse colonic inflammation was stimulated by introducing $2.5 \%$ DSS in drinking water. DSS treatment elevates cytokines and triggers the infiltration of inflammatory cells in the colon. ${ }^{33,34}$ Strong nuclear staining of Foxo3 was detected in mouse colonic epithelial cells, and slight cytosolic staining was found in crypt cells. ${ }^{21}$ In DSS-treated mice, Foxo3 was detected primarily in the cytosol of the colonic epithelia with no nuclear staining (Figure 6a and b), suggesting that Foxo3 is not active in the inflamed colonic epithelia. These findings correlate with in vitro data where Foxo3 translocation into the cytosol was detected. The degradation of Foxo3 in vivo was not apparent; we hypothesize that in intestinal tissue Foxo3 may

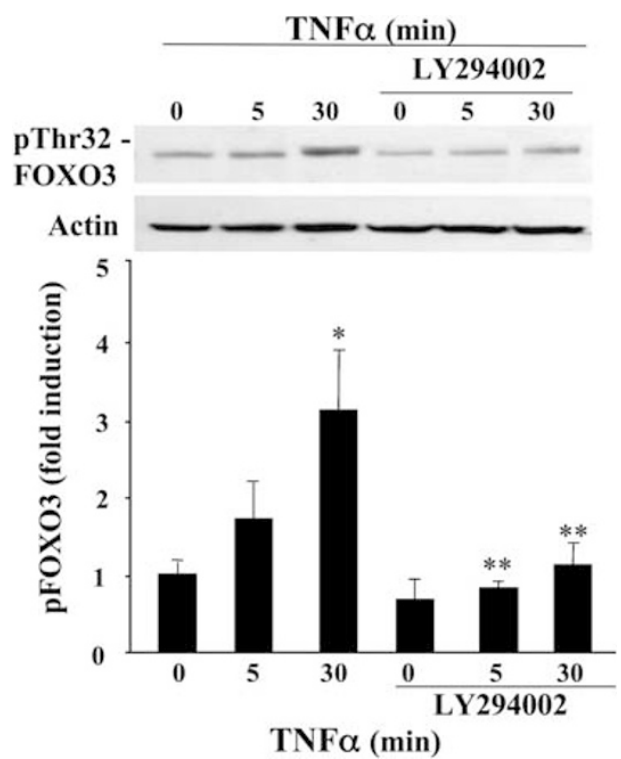

Figure $3 \mathrm{TNF} \alpha$-induced FOXO3 phosphorylation is $\mathrm{PI} 3 \mathrm{~K}$ dependent. The HT-29 cells; with or without pretreatment with PI3K inhibitor (LY294002) were incubated with TNF $\alpha$. Protein was separated on SDS-PAGE and immunoprobed with an antibody against phosphorylated FOXO3 at the Thr32 PI3K-dependent site and actin. The immunoblot is representative of three independent experiments (three samples were used per experimental group). Densitometric analysis shows a significant increase $\left(^{*}\right)$ in phosphorylated FOXO3 after TNF $\alpha$ treatment, which is attenuated in the presence of LY294002 ${ }^{(*)}(P<0.05)$.

also degrade but at this particular time point of DSS treatment degradation was not noticeable.

\section{Foxo3 Deficiency Leads to Increased Inflammation in the DDS Model}

To further assess the role of Foxo3 in intestinal inflammation in vivo, the Foxo3-deficient mice were employed. Although Lin reported that these animals displayed spontaneous inflammation in several organs, ${ }^{20}$ the intestinal tissue did not show signs of inflammation over a period of 2 months (Figure 7c). Wild-type and Foxo3-deficient mice from the same colony, treated with DSS, did not show significant differences in weight loss (WT: $21 \pm 4 \mathrm{~g}, \mathrm{KO}: 21 \pm 2 \mathrm{~g}$ ); however, there was increased blood in the stool in Foxo3deficient mice (Figure 7a) suggesting that the inflammatory process might be more active. A histological examination showed that the injury was significantly less severe and the recovery was enhanced in the wild-type mice relative to Foxo3-deficient mice. Although mild active inflammation was present in the wild-type mice, severe inflammation was present in the Foxo3-deficient mice (Figure 7b). The ulceration was minimal in wild-type mice, involved small areas, and was accompanied by evidence of mucosal healing (Figure 7c). In contrast, there were broad areas of mucosal ulceration with only limited healing present in Foxo3deficient mice. In Foxo3-deficient mice the lamina propria was expanded with a mixed population of lymphocytes and polymorphonuclear neutrophils (PMNs), especially in 


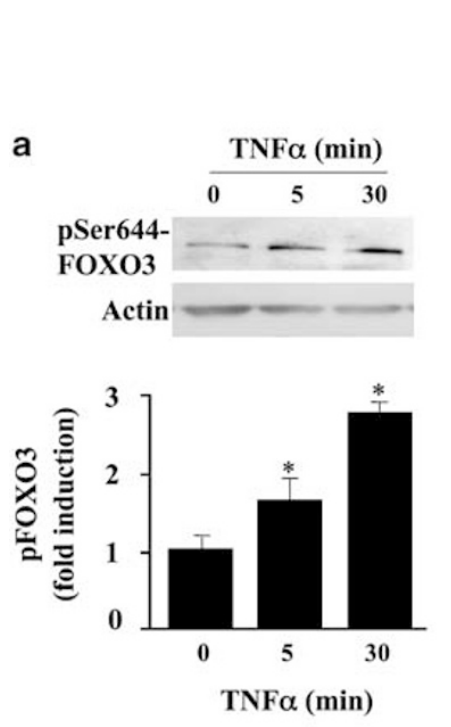

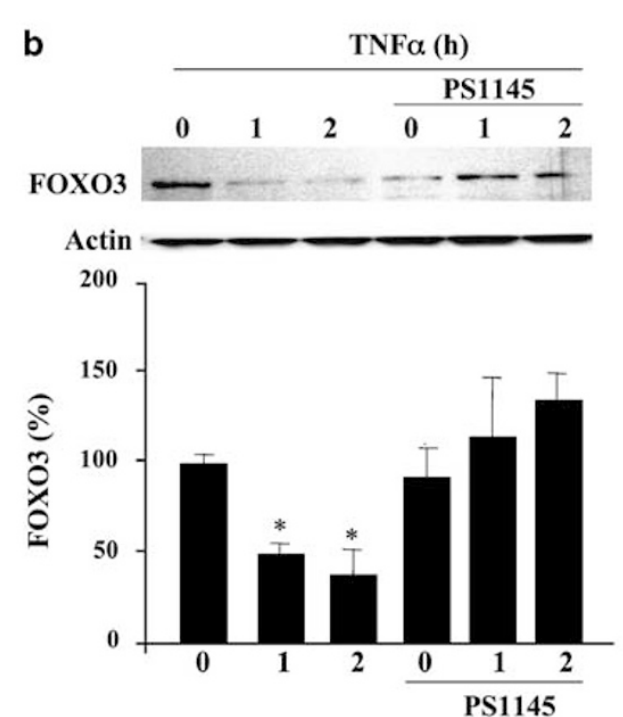

TNF $\alpha(h)$
C
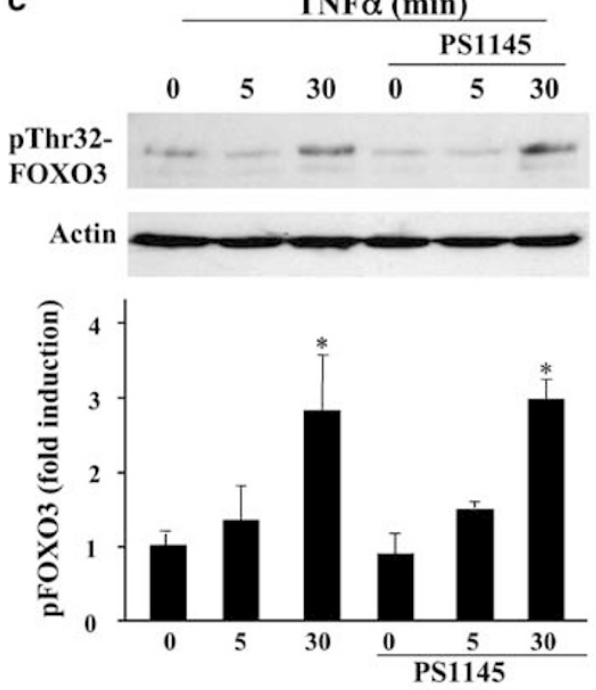

$\mathrm{TNF} \alpha(\min )$

Figure 4 TNF $\alpha$-induced inactivation of FOXO3 is controlled by IKK. (a) Total proteins from untreated and TNF $\alpha$-treated cells were separated on SDS-PAGE and immunoprobed with an antibody against phosphorylated FOXO3 at the Ser644 IKK-dependent position. Immunoblots were also re-probed with an antibody against actin. (b) The HT-29 monolayers were pre-treated with the IKK inhibitor, PS1145, and induced with TNF $\alpha$ for various time points. Protein was separated on SDS-PAGE and immunoprobed with an antibody against total FOXO3 and actin. The graphs represent the densitometric analysis showing a significant decrease of FOXO3 $\left.{ }^{*}\right)$ after TNF $\alpha$ treatment $(n=3, P<0.05)$ and protection of degradation with the IKK inhibitor. (c) Protein from the monolayers pretreated with PS1145 and TNF $\alpha$ was separated and immunoprobed against phosphorylated FOXO3 at Thr32 PI3K-dependent site and actin. The densitometric analysis shows a significant increase ${ }^{*}$ ) in phosphorylated FOXO3 after TNF $\alpha$ treatment, which was not attenuated in the presence of PS1145.
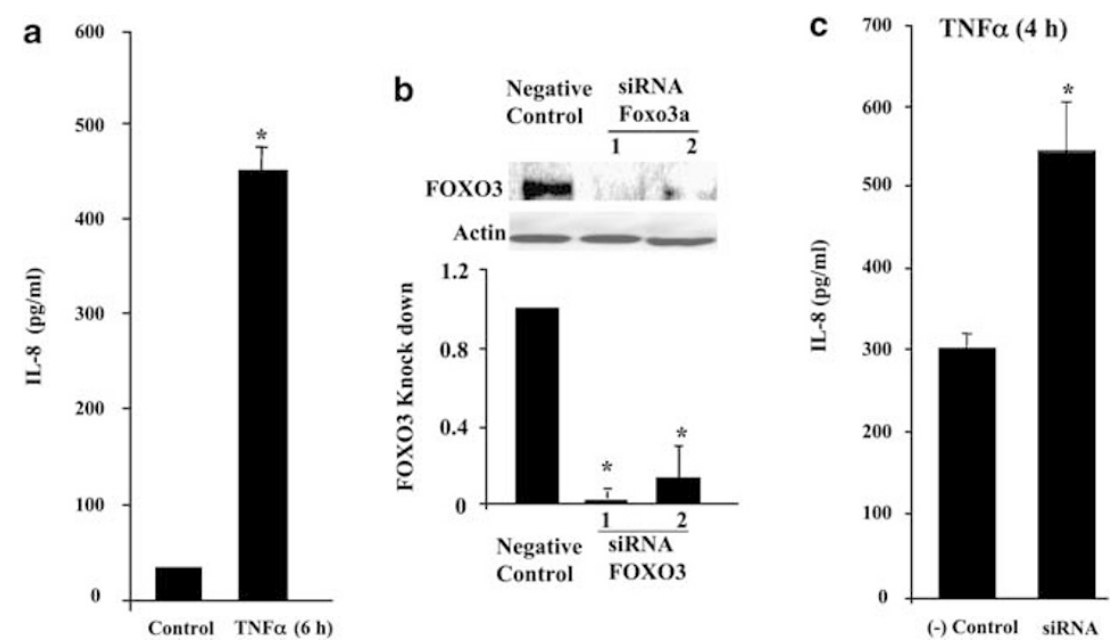

Figure 5 FOXO3 is involved in the regulation of TNF $\alpha$-induced IL-8 expression. (a). The monolayers were treated with TNF $\alpha$ for a period of $6 \mathrm{~h}$ and media was collected for IL-8 quantification. (b) Representative immunoblot of three independent experiments showing efficiency of FOXO3 knock down. The densitometric analysis shows significant ${ }^{*}$ ) knock down of FOXO3 after $48 \mathrm{~h}, n=3, P<0.05$. (c) The monolayers with silent FOXO3 were treated with TNF $\alpha$ for $4 \mathrm{~h}$ and media was collected for IL-8 quantification. The graph represents the average IL-8 ratio of three independent experiments and the asterisk represents a significant difference $(n=4, P<0.05)$.

areas close to ulcerations. In the intestinal epithelia, the number of lymphocytes and PMNs was increased 2- and 5-fold, respectively (lymphocytes: WT $4.1 \pm 1.4 ; \mathrm{KO} 7.6 \pm 3.2$, PMN: WT $0.9 \pm 1.1$; KO $6.4 \pm 2.7$ ) (Figure $7 \mathrm{~d}$ and e). These data show that Foxo3 deficiency results in severe disease in response to DSS.

\section{DISCUSSION}

In the pathogenesis of inflammatory diseases, cytokines have an important role in maintaining the inflammatory response. The regulation of cytokines is better understood today; however, further elucidation of the mechanisms of cytokine regulation is needed to develop more specific and effective 
a

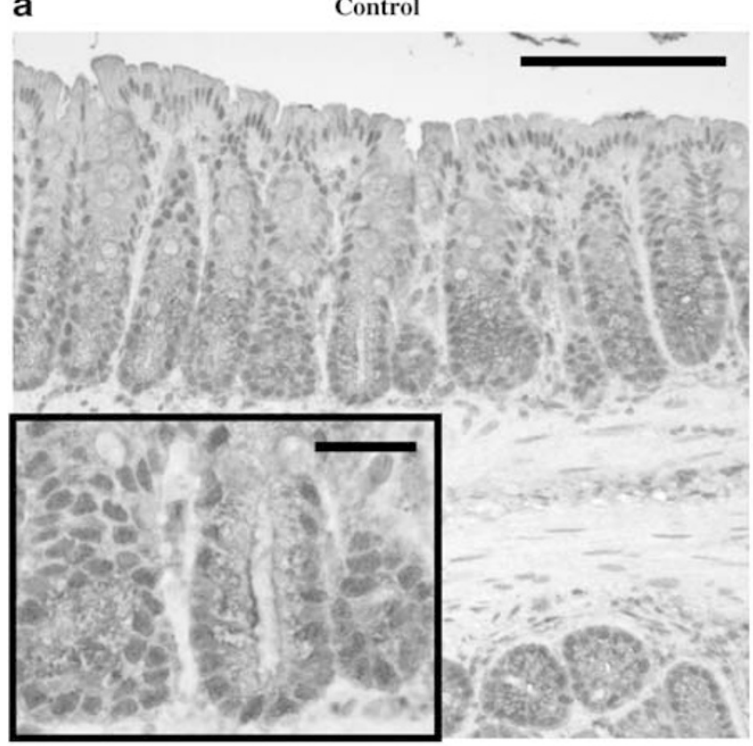

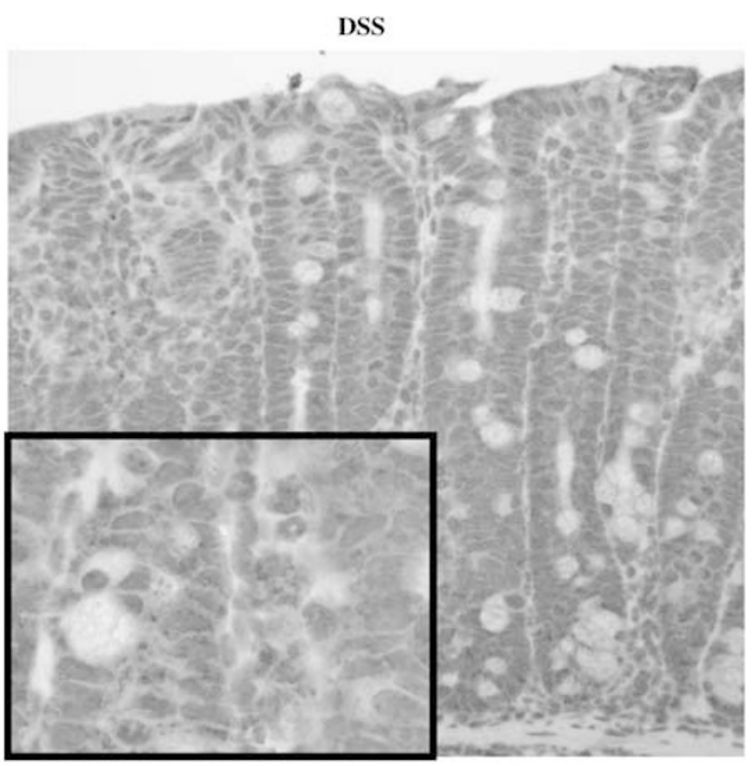

\section{b}

WT
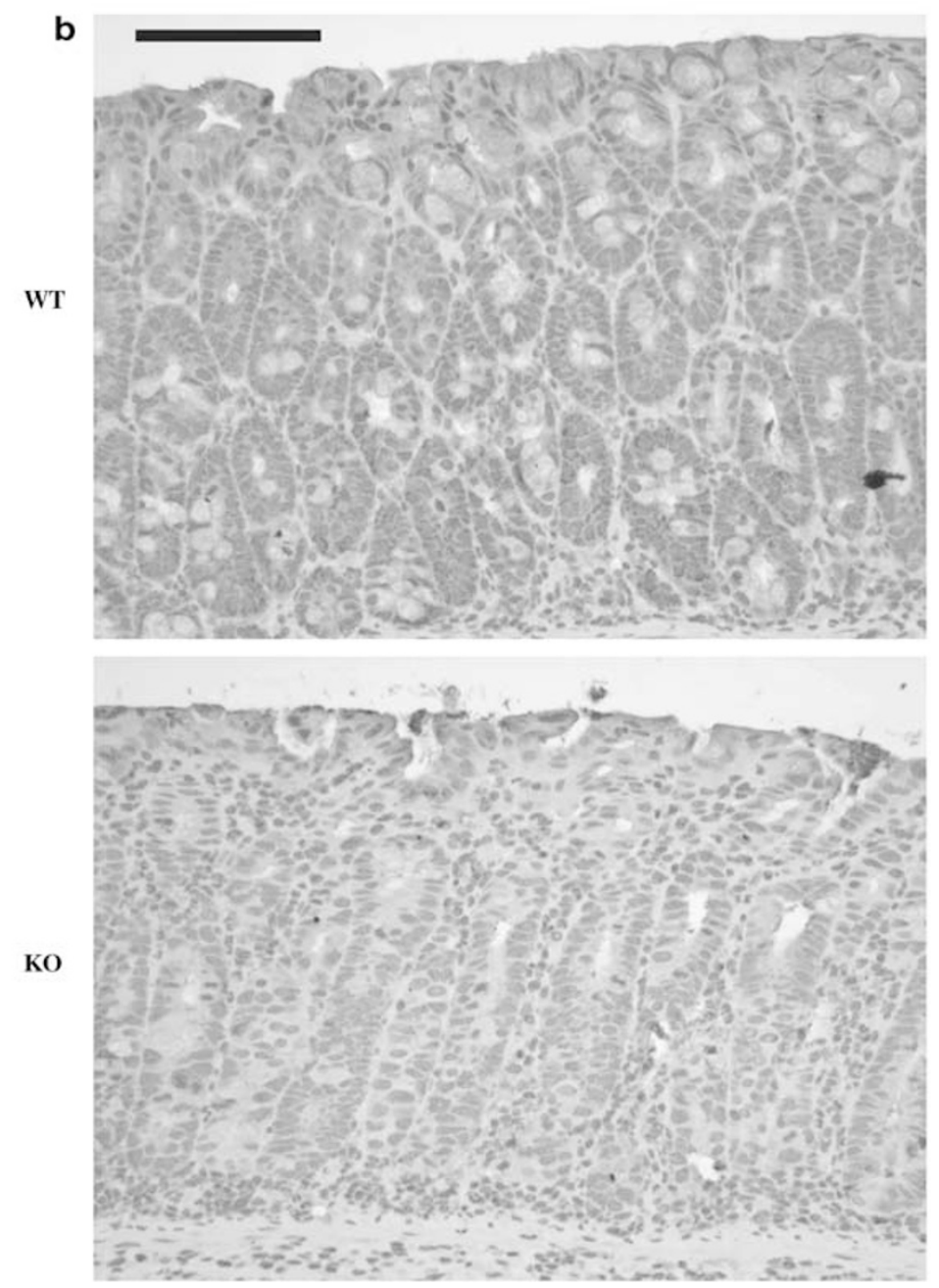

Figure 6 Foxo3 status in colonic epithelia of mice with DSS-induced inflammation. (a) Colonic tissue from C57BL/J6 mice, control and treated with DSS were immunohistostained for Foxo3. Immunohistostaining revealed cytoplasmic Foxo3 localization in inflamed colonic epithelia. (b) Colonic tissue from Foxo3-deficient mice is immunohistostained for Foxo3 as a control ( $\times 20$ magnification: bar $100 \mu \mathrm{m}$; inset $\times 63$ magnification: bar $40 \mu \mathrm{m}$ ). 

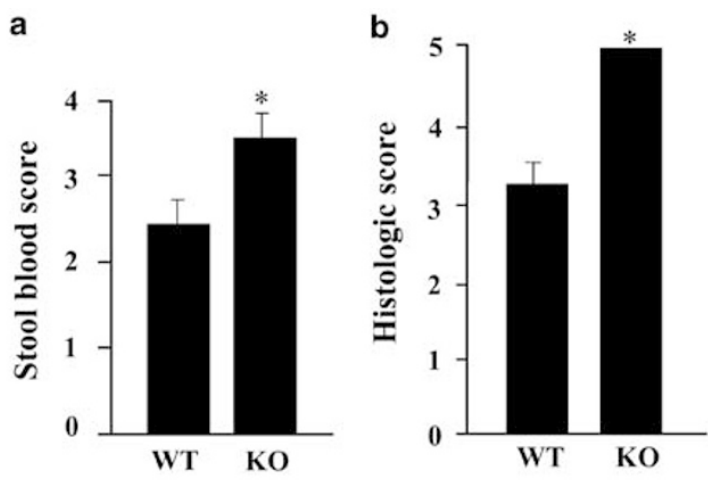

C

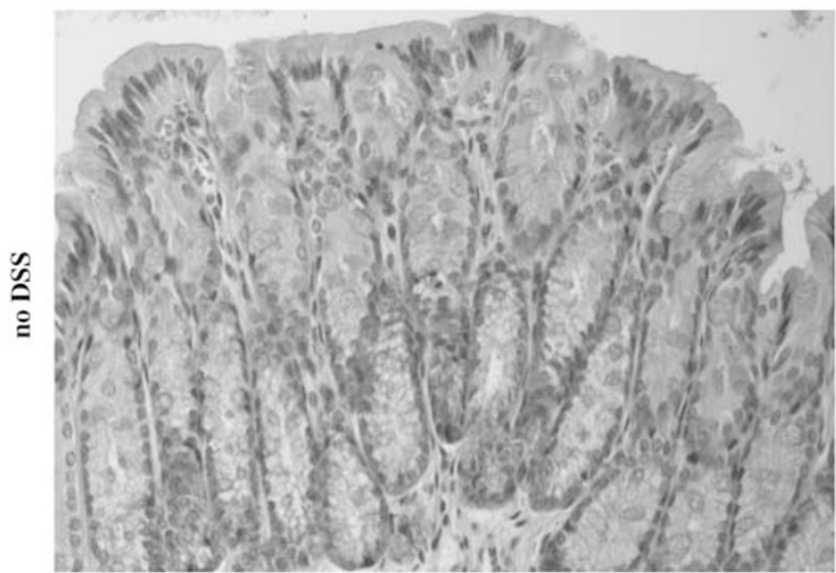

WT

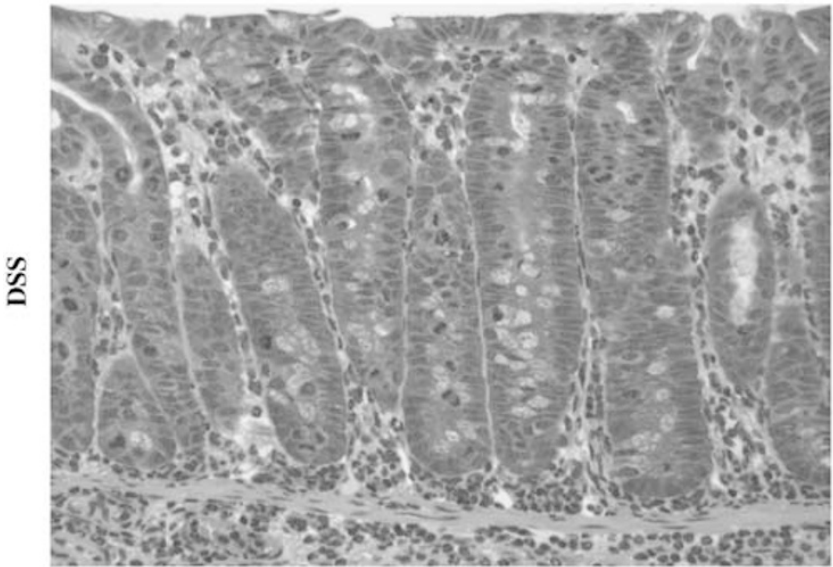

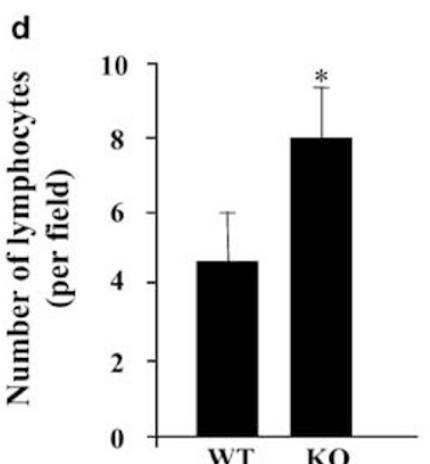

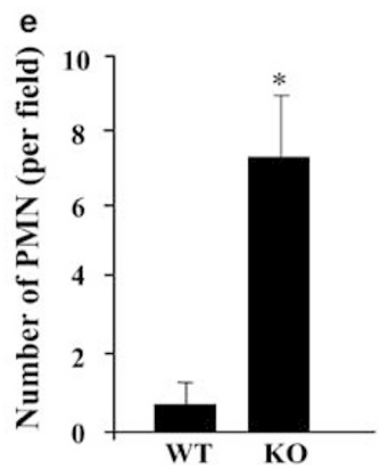

KO
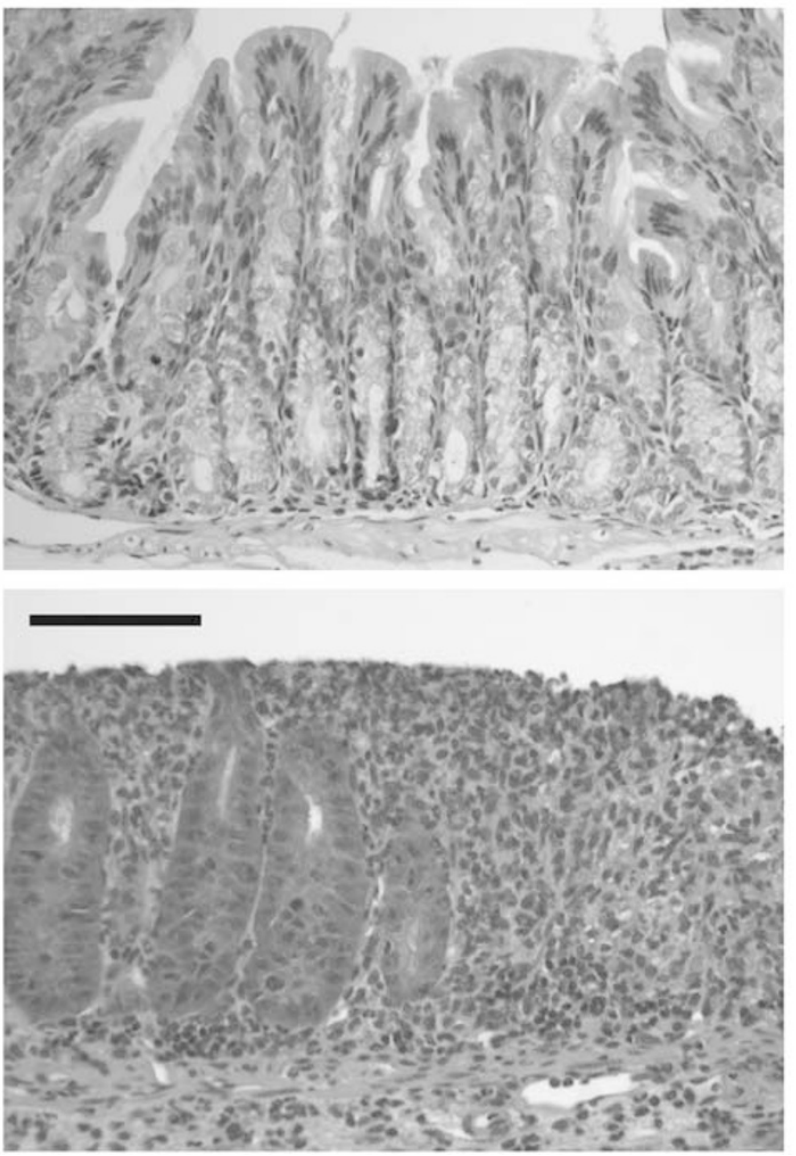

Figure 7 Foxo3 deficiency leads to severe intestinal inflammation. Foxo3 knockout (KO) and wild-type (WT) mice ( $n=7$ from each group) were treated with $2.5 \%$ DSS for 5 days and left 2 days to recover. (a) Measuring blood in stool revealed that KO mice have more bleeding than WT mice ( $P<0.05$ ). (b) Graph

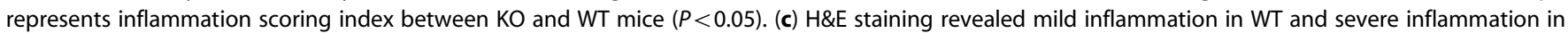
KO colon ( $\times 20$ magnification: bar $100 \mu \mathrm{m}$ ). (d and e) Graphs represent the number of lymphocytes and PMNs in colonic epithelia enumerated in five different fields. Asterisk represents significant differences between WT and KO mice $(P<0.05)$.

therapies. Here, we have shown that TNF $\alpha$ induces FOXO3 phosphorylation, translocation, and degradation in HT-29 cells. TNF $\alpha$-mediated FOXO3 degradation is proteasome dependent. Proximally, TNF $\alpha$ used the PI3K and IKK pathways to regulate FOXO3 in HT-29 cells. In addition, our in vitro data revealed that silenced FOXO3 leads to drama- tically increased TNF $\alpha$-induced IL- 8 expression in HT-29 cells, which is in agreement with our previously published data. ${ }^{21}$ In vivo, in the mouse DSS colitis model, Foxo3 is localized primarily in the cytosol of the intestinal epithelia, suggesting that Foxo3 is inactive. Also, Foxo3-deficient mice developed a more severe intestinal inflammation compared 
with wild-type mice in response to the DSS treatment. Altogether, our results suggest that tumor suppressor FOXO3 regulates intestinal inflammation in vitro and in vivo.

FOXO3 is localized in the nucleus-controlling gene expression both directly and indirectly. ${ }^{13}$ In certain tumor cells, the inactivated cytosolic FOXO3 contributes to the pathogenesis and development of tumors by modulating proliferation and apoptosis. ${ }^{35-37}$ Our in vivo data revealed that Foxo3 is localized in the cytosol in the inflamed colon and that Foxo3 deficiency additionally promotes the inflammation. We previously demonstrated that FOXO3 knock down leads to the reduced inhibitory $\mathrm{I} \kappa \mathrm{B} \alpha$ in intestinal epithelial cells. ${ }^{21}$ Therefore, we concluded that the inactive cytosolic $\mathrm{FOXO} 3$ further promotes intestinal inflammation. Kristof $e t a l^{38}$ have shown that LPS controls FOXO3 in human lung epithelial cells, thus regulating iNOS and contributing to inflammation. In macrophages, HIV infection keeps FOXO3 in the nucleus, which further contributes to the apoptosis of infected cells. ${ }^{39}$ Thus FOXO3 has very different, but important, roles in the regulation of cellular events depending on the cell type and pathological conditions.

FOXO3 is controlled proximally by the PI $3 \mathrm{~K}$ and IKK pathways. ${ }^{13,15}$ Our data show that TNF $\alpha$ regulates FOXO3 by both PI3K and IKK pathways in human intestinal HT-29 cells. Previously, we have shown that bacterial infection regulates FOXO3 via PI3K, whereas the role of IKK was insignificant. ${ }^{21}$ Thus it appears that the proximal pathways differ depending on the stimulus. At this moment, the exact relationship between the PI3K and IKK pathways, with regard to $\mathrm{TNF} \alpha$-dependent $\mathrm{FOXO} 3$ regulation in intestinal epithelial cells, is unclear. The inhibition of IKK did not affect PI3Kdependent $\mathrm{FOXO} 3$ phosphorylation, whereas we were unable to clarify how inhibition of PI3K affects IKK-dependent FOXO3 phosphorylation. We speculate that the PI3K and IKK pathways regulate FOXO3 independently. Sudheerkumar et $a l^{40}$ have demonstrated that TNF $\alpha$ can independently activate IKK and PI3K pathways in human glioma cells. Agarwal et $a l^{41}$ showed that simultaneous activation of the IKK and PI3K pathways regulate both NF- $\kappa \mathrm{B}$ and $\beta$-catenin to facilitate tumor progression. We hypothesize that TNF $\alpha$ facilitates the inflammation of the intestinal epithelia by activating the PI3K and IKK pathways that regulate both $\mathrm{NF}-\kappa \mathrm{B}$ and $\mathrm{FOXO} 3$. We speculate that $\mathrm{TNF} \alpha$ activates two separate but interrelated signaling pathways and that both are necessary for the full inactivation of FOXO3, which further promotes inflammation. One set of signals activates the IKK complex to phosphorylate FOXO3 and to initially liberate $\mathrm{NF}-\kappa \mathrm{B}$ and drive the immediate cytokine expression. The second set of signals uses the PI3K mechanism to additionally phosphorylate FOXO3 and keep it out of the nucleus so that the de novo $\mathrm{I} \kappa \mathrm{B} \alpha$ synthesis is repressed and the transcription of pro-inflammatory genes is uninterrupted.

Foxo3 deficiency in mice leads to spontaneous T-cell activation, cytokine production and a mild lymphoprolifera- tion. In Foxo3-deficient mice, this leads to an autoimmune syndrome with spontaneous inflammation in multiple organs, while having no reported intestinal changes. ${ }^{20}$ The intestinal epithelia have an important role in separating the unsterile from the sterile environment and thus, the infiltration of immune cells is tightly controlled. Intestinal inflammation might occur after the intestinal epithelia 'sense' the environmental changes and send signals to summon immune cells to infiltrate the tissue. Therefore, we speculate that the role of Foxo3 in the intestinal tissue is specific. In an inflamed colon, Foxo3 is distributed in the cytosol of the intestinal epithelia, suggesting that Foxo3 is inactive. We hypothesize that the localization of $\mathrm{FOXO} 3$ will correlate with the severity of inflammation in the DSS model and that with mild inflammation, Foxo3 will be distributed between the cytosol and the nucleus. In cultured intestinal cells TNF $\alpha$ induces cytosolic FOXO3 degradation, which was not apparent in the intestinal epithelia of the colon. The intestinal epithelial cells in tissue permanently proliferate and differentiate, which is critical for normal growth, development, and disease prevention. FOXO3 also regulates the proliferation of these cells, thus we hypothesize that $\mathrm{FOXO} 3$ remains in the cytosol without significant degradation and is ready to go back to the nucleus to regulate other functions.

Foxo3-deficient mice develop more severe inflammatory responses to DSS compared with wild-type mice. We show in vitro that $\mathrm{FOXO} 3$ deficiency leads to the attenuation of inhibitory $\mathrm{I} \kappa \mathrm{B} \alpha,{ }^{21}$ directly linking $\mathrm{FOXO} 3$ with inflammation. A similar role of Foxo3 was observed in T cells. In mouse $\mathrm{T}$ cells deficient in Foxo3, NF- $\kappa \mathrm{B}$ activation is unrestrained and there are diminished levels of I $\kappa \mathrm{Bs} .^{20}$ It is possible that for colonic inflammation in the DSS model, lymphocytes deficient in Foxo3 are in part responsible. However, the fact that Foxo3-deficient lymphocytes alone do not spontaneously infiltrate colonic tissue suggest that Foxo3 has the primary role in intestinal epithelia. In B cells and PMNs, Foxo3 has less of an effect on the NF- $\kappa$ B pathway, but it does regulate cell survival and proliferation. ${ }^{42,43}$ Foxo3-deficient mice were resistant to induced arthritis due to increased apoptosis of the Foxo3-deficient PMNs. ${ }^{44}$ On the contrary, our data showed that in colonic epithelia PMN accumulation and crypt abscesses are increased in Foxo3-deficient mice. We proposed two possible scenarios: (a) infiltrated Foxo3-deficient PMNs in the intestinal tissue are resistant to apoptosis; and (b) due to the large number of PMNs infiltrated in the colon the apoptotic nature on Foxo3-deficient PMN cells is not enough to eliminate them. Yet the role of Foxo3 in infiltrated inflammatory cells in the colon is still unclear as well as FOXO3's role in the healing of inflamed intestinal tissue. We need to further address these questions.

In summary, these data indicate that FOXO3 has an important role in controlling and facilitating intestinal inflammation. Furthermore, FOXO3 should be considered as a potential therapeutic target to treat IBD. 


\section{ACKNOWLEDGEMENTS}

We thank Dr Hemant Roy, Dr Michael Goldberg, and Dr Ramesh Wali for their helpful assistance in preparation of the article. We also thank Dr Stanford Peng (Roche Palo Alto LLC, Palo Alto, CA, USA) for providing the Foxo3-deficient mice to establish our breeding colony. This work was in part supported by a Senior Investigator Award from the Crohn's and Colitis Foundation of America (CCFA no. 1953) and North Shore University Health System Pilot Study grant. Lobke Snoeks received a Fulbright scholarship for her visiting fellowship and Christopher Weber received an $\mathrm{NIH}$ F32DK082134 fellowship award.

\section{DISCLOSURE/CONFLICT OF INTEREST}

The authors declare no conflict of interest.

1. Podolsky DK. Inflammatory bowel disease. New Eng J Med 2002;347:417-429.

2. Breese EJ, Michie CA, Nicholls SW, et al. Tumor necrosis factor alphaproducing cells in the intestinal mucosa of children with inflammatory bowel disease. Gastro 1994;106:1455-1466.

3. Maeda $\mathrm{M}$, Watanabe $\mathrm{N}$, Neda $\mathrm{H}$, et al. Serum tumor necrosis factor activity in inflammatory bowel disease. Immunopharm Immunotox 1992;14:451-461.

4. Komatsu M, Kobayashi D, Saito K, et al. Tumor necrosis factor-alpha in serum of patients with inflammatory bowel disease as measured by a highly sensitive immuno-PCR. Clin Chem 2001;47:1297-1301.

5. van Dullemen HM, van Deventer SJ, Hommes DW, et al. Treatment of Crohn's disease with anti-tumor necrosis factor chimeric monoclonal antibody (cA2). Gastro 1995;109:129-135.

6. Reid T, Louie P, Heller RA. Mechanisms of tumor necrosis factor cytotoxicity and the cytotoxic signals transduced by the p75-tumor necrosis factor receptor. Circ Shock 1994;44:84-90.

7. Kaiser GC, Polk DB. Tumor necrosis factor alpha regulates proliferation in a mouse intestinal cell line. Gastro 1997;112:1231-1240.

8. Brockhaus M, Schoenfeld HJ, Schlaeger EJ, et al. Identification of two types of tumor necrosis factor receptors on human cell lines by monoclonal antibodies. Proc Nat Acad Sci USA 1990;87:3127-3131.

9. Kruppa G, Thoma B, Machleidt $T$, et al. Inhibition of tumor necrosis factor (TNF)-mediated NF- $\kappa$ B activation by selective blockade of the human 55-kDa TNF receptor. J Immunol 1992;148:3152-3157.

10. Tak PP, Firestein GS. NF- $\kappa$ B: a key role in inflammatory diseases. J Clin Invest 2001;107:7-11.

11. Cortes Sempere M, Rodriguez Fanjul V, Sanchez Perez I, et al. The role of the NF- $\kappa$ B signalling pathway in cancer. Clin Transl Oncol 2008:10:143-147.

12. Schottelius AJ, Dinter H. Cytokines, NF- $\kappa$ B, microenvironment, intestinal inflammation and cancer. Canc Treat Res 2006;130:67-87.

13. Burgering BM, Kops GJ. Cell cycle and death control: long live forkheads. Trends Biochem Sci 2002;27:352-360.

14. Alvarez $B$, Martinez AC, Burgering BM, et al. Forkhead transcription factors contribute to execution of the mitotic programme in mammals. Nature 2001;413:744-747.

15. Hu MC, Lee DF, Xia W, et al. $1 \kappa \mathrm{B}$ kinase promotes tumorigenesis through inhibition of forkhead FOXO3a. Cell 2004;117:225-237.

16. Birkenkamp KU, Coffer PJ. FOXO transcription factors as regulators of immune homeostasis: molecules to die for? J Immunol 2003;171:1623-1629.

17. Yaffe $M B$, Rittinger $K$, Volinia $S$, et al. The structural basis for 14-3-3:phosphopeptide binding specificity. Cell 1997;91:961-971.

18. Kops GJ, de Ruiter ND, De Vries-Smits AM, et al. Direct control of the Forkhead transcription factor AFX by protein kinase B. Nature 1999;398:630-634.

19. Aoki M, Jiang H, Vogt PK. Proteasomal degradation of the FoxO1 transcriptional regulator in cells transformed by the P3K and Akt oncoproteins. Proc Nat Acad Sci USA 2004;101:13613-13617.

20. Lin L, Hron JD, Peng SL. Regulation of NF- $\kappa$ B, Th activation, and autoinflammation by the forkhead transcription factor Foxo3a. Immunity 2004;21:203-213.

21. Snoeks $L$, Weber CR, Turner JR, et al. Tumor suppressor Foxo3a is involved in the regulation of LPS-induced IL-8 in intestinal HT-29 cells. Infect Immun 2008;76:4677-4685.
22. Vodanovic-Jankovic $\mathrm{S}$, Hari $\mathrm{P}$, Jacobs $\mathrm{P}$, et al. NF-kappaB as a target for the prevention of graft-vs-host disease: comparative efficacy of bortezomib and PS-1145. Blood 2006;107:827-834.

23. Yemelyanov A, Gasparian A, Lindholm P, et al. Effects of IKK inhibitor PS1145 on NF- $\kappa$ B function, proliferation, apoptosis and invasion activity in prostate carcinoma cells. Oncogene 2006;25:387-398.

24. Sakurai $\mathrm{H}$, Chiba $\mathrm{H}$, Miyoshi $\mathrm{H}$, et al. $I \kappa \mathrm{B}$ kinases phosphorylate NF- $\kappa \mathrm{B}$ p65 subunit on serine 536 in the transactivation domain. J Biol Chem 1999;274:30353-30356.

25. Larsen CG, Anderson AO, Oppenheim JJ, et al. Production of interleukin- 8 by human dermal fibroblasts and keratinocytes in response to interleukin-1 or tumour necrosis factor. Immunology 1989;68:31-36.

26. Kasahara T, Mukaida N, Yamashita $\mathrm{K}$, et al. IL-1 and TNF $\alpha$ induction of IL-8 and monocyte chemotactic and activating factor (MCAF) mRNA expression in a human astrocytoma cell line. Immunology 1991;74:60-67.

27. Uguccioni $M$, Gionchetti $P$, Robbiani DF, et al. Increased expression of IP-10, IL-8, MCP-1, and MCP-3 in ulcerative colitis. Am J Pathol 1999;155:331-336.

28. McLaughlan JM, Seth R, Vautier G, et al. Interleukin-8 and inducible nitric oxide synthase mRNA levels in inflammatory bowel disease at first presentation. J Pathol 1997;181:87-92.

29. Izzo RS, Witkon $\mathrm{K}$, Chen $\mathrm{Al}$, et al. Neutrophil-activating peptide (interleukin-8) in colonic mucosa from patients with Crohn's disease. Scand J Gastro 1993;28:296-300.

30. Mazzucchelli L, Hauser C, Zgraggen K, et al. Expression of interleukin-8 gene in inflammatory bowel disease is related to the histological grade of active inflammation. Am J Pathol 1994;144:997-1007.

31. Eckmann L, Jung HC, Schurer-Maly C, et al. Differential cytokine expression by human intestinal epithelial cell lines: regulated expression of interleukin 8. Gastro 1993;105:1689-1697.

32. Eckmann L, Kagnoff MF, Falco MT. Colonic epithelial cell lines as a source of interleukin-8: stimulation by inflammatory cytokines and bacterial lipopolysaccharide. Immunology 1994;82:505.

33. Herfarth $\mathrm{H}$, Brand $\mathrm{K}$, Rath $\mathrm{HC}$, et al. Nuclear factor- $\kappa \mathrm{B}$ activity and intestinal inflammation in dextran sulphate sodium (DSS)-induced colitis in mice is suppressed by gliotoxin. Clin Exp Immun 2000;120: $59-65$.

34. Saubermann LJ, Nakajima A, Wada K, et al. Peroxisome proliferatoractivated receptor gamma agonist ligands stimulate a Th2 cytokine response and prevent acute colitis. Inflamm Bow Dis 2002;8:330-339.

35. Nicholson KM, Anderson NG. The protein kinase B/Akt signalling pathway in human malignancy. Cell Signal 2002;14:381-395.

36. Medema RH, Kops GJ, Bos JL, et al. AFX-like Forkhead transcription factors mediate cell-cycle regulation by Ras and PKB through p27kip1. Nature 2000;404:782-787.

37. Nakamura N, Ramaswamy S, Vazquez F, et al. Forkhead transcription factors are critical effectors of cell death and cell cycle arrest downstream of PTEN. Mol Cell Biol 2000;20:8969-8982.

38. Kristof AS, Fielhaber J, Triantafillopoulos $A$, et al. Phosphatidylinositol 3-kinase-dependent suppression of the human inducible nitric-oxide synthase promoter is mediated by FKHRL1.J Biol Chem 2006;281:23958-23968.

39. Cui $M$, Huang $Y$, Zhao $Y$, et al. Transcription factor FOXO3a mediates apoptosis in HIV-1-infected macrophages. J Immunol 2008;180:898-906.

40. Sudheerkumar P, Shiras A, Das G, et al. Independent activation of Akt and $\mathrm{NF}-\kappa \mathrm{B}$ pathways and their role in resistance to $\mathrm{TNF} \alpha$ mediated cytotoxicity in gliomas. Mol Carc 2008;47:126-136.

41. Agarwal A, Das K, Lerner N, et al. The AKT/l $\kappa B$ kinase pathway promotes angiogenic/metastatic gene expression in colorectal cancer by activating NF- $\kappa \mathrm{B}$ and $\beta$-catenin. Oncogene 2005:24:1021-1031.

42. Jacobsen $E A$, Ananieva O, Brown ML, et al. Growth, differentiation, and malignant transformation of pre-B cells mediated by inducible activation of v-Abl oncogene. J Immunol 2006;176:6831-6838.

43. Crossley LJ. Neutrophil activation by fMLP regulates FOXO (forkhead) transcription factors by multiple pathways, one of which includes the binding of FOXO to the survival factor Mcl-1. J Leukoc Biol 2003;74:583-592.

44. Jonsson H, Allen P, Peng SL. Inflammatory arthritis requires Foxo3a to prevent Fas ligand-induced neutrophil apoptosis. Nature Med 2005;11:666-671. 